\title{
Arthroscopic Debridement with Lavage and Arthroscopic Lavage Only as the Treatment of Symptomatic Osteoarthritic Knee
}

\author{
Suhail Karkabi, Nahum Rosenberg \\ Shoulder Service, Rambam Health Care Campus, Haifa, Israel \\ Email: $\underline{\text { skarkabi@rambam.health.gov.il }}$
}

Received 25 February 2015; accepted 5 June 2015; published 8 June 2015

Copyright (C 2015 by authors and Scientific Research Publishing Inc.

This work is licensed under the Creative Commons Attribution International License (CC BY). http://creativecommons.org/licenses/by/4.0/

(c) (i) Open Access

\begin{abstract}
In this prospective follow up study we evaluated and compared the effect of arthroscopic lavage with and without debridement as a treatment of moderately osteoarthritic knees in middle aged and elderly population. The study group comprised of 500 patients $(250$ patients for each type of treatment, mean age 58 years). The patients were followed six months, three years and six years postoperatively. Following both treatments there was a clear deterioration in function of the knee function over the time, as measured starting at six months follow up until six years postoperatively $(p<0.001)$. This deterioration was more prominent in patients after treatment by the arthroscopic lavage only. We show that for the patients with high functional demand and expectations for long term rehabilitation and improvement both arthroscopic treatments are not satisfactory in more of $60 \%$ of cases.
\end{abstract}

\section{Keywords}

Arthroscopy, Knee, Osteoarthritis, Debridement, Lavage

\section{Introduction}

Degenerative arthritis (Osteoarthritis, OA) of the knee is a common cause of knee pain. The pain from osteoarthritis is due to synovitis, capsular and ligamentous inflammation, and subchondral bone edema.

$\mathrm{OA}$ is commonly the end result of abnormally distributed mechanical stress on the articular cartilage, either through a sudden single load or following a cumulative effect of multiple and/or repetitive loads that lead to mechanical disintegration of the structure of hyaline cartilage.

The treatment of knee pain due to OA is usually directed to oppose the causative abnormal articular mechanics, 
e.g. conservative treatment by rest, weight loss, physical therapy for strengthening thigh musculature and subsequentially reducing intrarticular forces; pharmacological treatment by nonsteroidal anti-inflammatory drugs or steroid injections in order to reduce local inflammation and surgical treatment, i.e. arthroscopic in order to debride superficial cartilage irregularities and induce new cartilage generation by deep subchondral exposure or aiming to correct the joint deformity by corrective osteotomy [1]-[3]. Occasionally visco supplementation therapy is used [4]. When the end stage OA is diagnosed, endoprosthetic arthroplasty is preferred.

Usually when the conservative options fail, arthroscopic debridement and lavage are rationally considered because of the less morbid outcome of this procedure [5].

Arthroscopic debridement of the degenerative knee is a common surgical procedure and could be beneficial in patients with symptomatic knee OA but without an extensively damaged articular surface. Arthroscopic debridement can be done as an outpatient procedure, because of its low postoperative morbidity. Following this type of surgery early return to the normal daily activities is expected. Therefore the rationale for offering arthroscopic debridement in the mild cases of knee OA is due to expected rapid improvement in symptoms and function without serious postoperative morbidity [6] [7]. From our clinical impression even less morbid procedure of arthroscopic knee joint lavage alone, without debridement, might be similarly beneficial in reducing symptoms in patients with mild but symptomatic knee OA. Therefore we hypothesized that arthroscopic lavage of knee should be as effective as arthroscopic intrarticular debridement in a selective group of patients with at the most moderate articulate degeneration.

\section{Methods}

This is a prospective follow up study. Between 1998 to 2007, five hundred patients underwent arthroscopic surgery for the treatment of knee OA .This patient group suffered from knee pain on effort, had radiographic evidence of minor to moderate joint space narrowing and marginal osteophyte formation (Figure 1). All these patients failed to respond to conservative treatment.

In this group 250 patients underwent arthroscopic debridement and lavage, and additional 250 patients underwent arthroscopic lavage only. The arthroscopic debridement included debridement and shaving of osteoarthritic articular cartilage and of degenerative meniscal lesions.

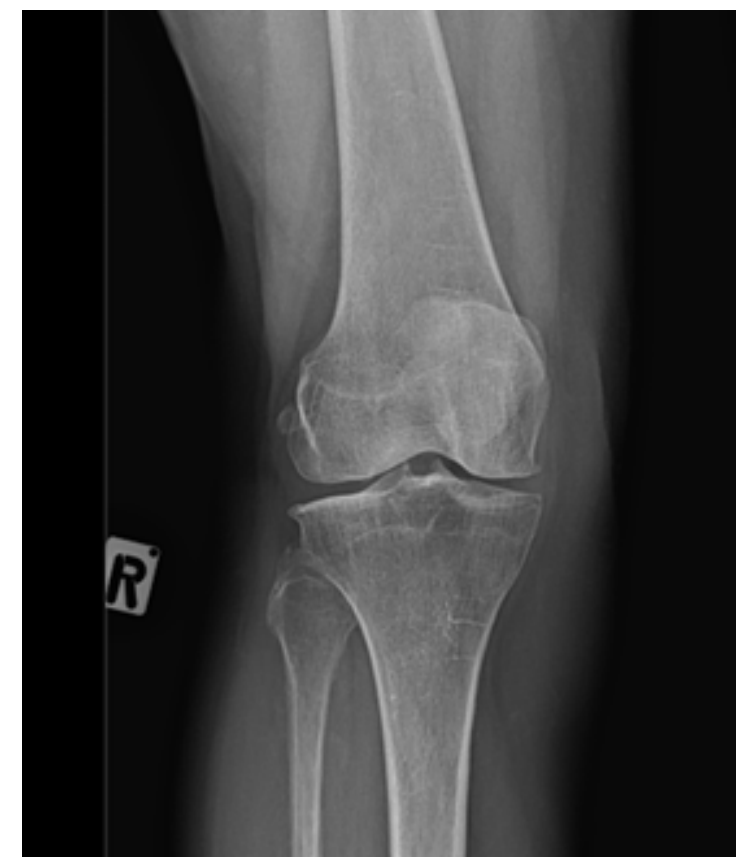

(a)

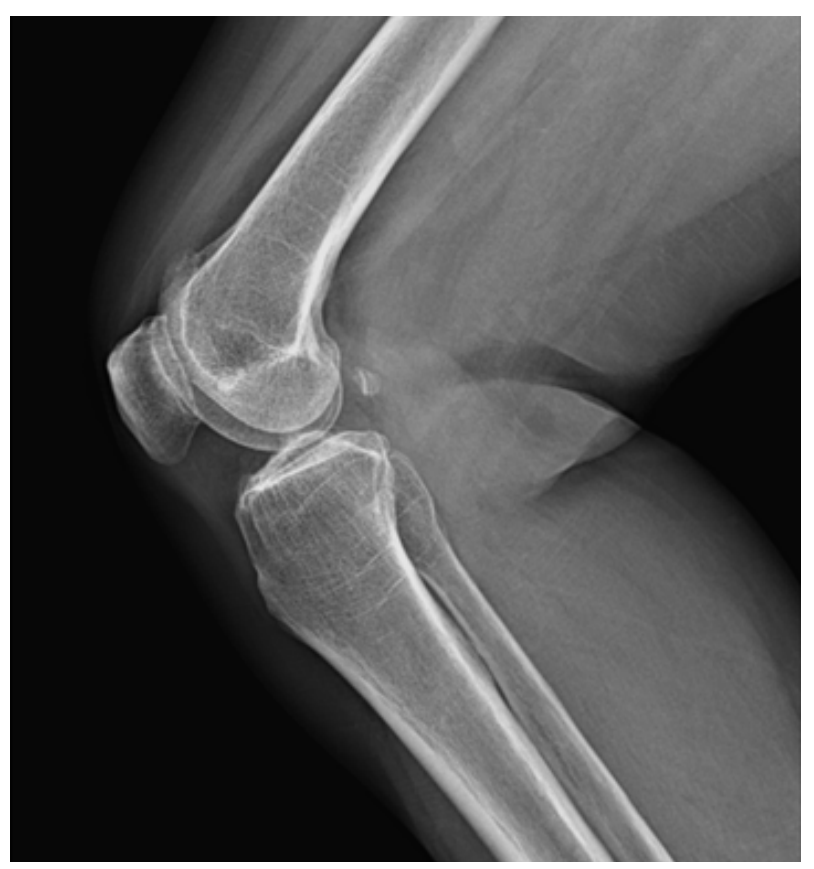

(b)

Figure 1. Representative radiographs of 69 years old women with moderate osteoarthritis of her right knee. Narrowing of the medial compartment, irregularities and sclerosis of the articular surface and small marginal osteophytes are apparent. (a) Anterior-posterior view; (b) Lateral view. 
Patients with inflammatory arthritis, severe misalignment of knee (valgus greater than 10 degrees or varus greater than 5 degrees), those with large meniscal tears or with radiographic evidence of the end stage OA and when a lack of full knee extension was evident were excluded from the study. The mean age of the patients at the time of surgery was 62 years (range, 52 to 78 years). For the purpose of this study each patient was evaluated six months after surgery and then after 3 years and six years postoperatively.

In all patients the arthroscopies were performed under local anesthesia by injecting through the routine portals into the knee joint of 10 cc of lidocaine $2 \%$ and 20 cc of bupivacaine $0.25 \%$. Tourniquet was not used. The arthroscopic findings, including the condition of articular cartilage, meniscal lesions and ligamentous insufficiency, were all recorded. All the surgeries were done by one of the authors (KS) and the study was approved by the Institutional Ethical Committee.

The modified Hospital for Special Surgery (HSS) scoring scale [8] was used as an objective scoring method for the preoperative and the postoperative knee symptoms. This score combines subjective an objective functional and mechanical components in 6 subscales, i.e. pain (30 points), walking and stairs (22 points), knee range of motion (18 points), deformity (10 points), instability (10 points). According to this score the knee function is defined as "excellent" when the score is above 85 points, "good" in the range of scores between 70 - 84 points, "fair” in the range of scores between 60 - 69 points and "poor" below 60 points.

The statistical comparison of the results was according to Chi Square test from contingency tables, $\mathrm{P}$ values below 0.05 determined the significant difference between the values [9].

\section{Results}

There were no deep infections or superficial wound problems, no deep vein thrombosis and no other major complications.

Twenty patients were lost to follow up at 6 months (9 from the "lavage only" group), additional 9 patients at 3 years (7 from the "lavage only" group) and 11 patients were lost to follow up at 6 years (5 from the "lavage only" group). Therefore $8 \%, 2 \%$, and $2 \%$ of patients were lost on the three stages of follow up respectively.

On initial short term postoperative follow up most of the patients from two treatment groups showed similar positive outcome, i.e. good and excellent knee function in $66 \%$ and $77 \%$ of patients after lavage only and debridement and lavage treatment respectively, with overall better outcome results in patients after debridement and lavage treatment ( $<<0.01$, Figure 2).

At midterm follow up, three years postoperatively, the outcome in the lavage only group was completely opposite to the initial short term evaluation, i.e. $69 \%$ of these patients showed poor knee function and only $21 \%$ of patients showed good and excellent function ( $<<0.001$, Figure 3 ). The midterm outcome in the patients after debridement and lavage was also worse than the their initial functional outcome but in lesser extent in comparison to the lavage only treatment, i.e. $38 \%$ of patients with good and excellent knee function and $44 \%$ of patients with poor function of the treated knee ( $<<0.001$, Figure 3$)$.

At long follow up of 6 years most of the patients in two groups had poor knee function, 84\% of patients after lavage treatment and $64 \%$ of patients after debridement and lavage treatment, but still with overall better outcome in patients of the latter group with almost double rate of good and excellent results, 25\% after debridement and lavage vs. $12 \%$ after lavage only group ( $\mathrm{p}<0.001$, Figure 4$)$.

Overall following both treatments there was a clear deterioration in function of the knee over the time, as measured starting at six months follow up to six years postoperatively ( $p<0.001$, Figure 5 ). This deterioration was more prominent in patients after treatment by the lavage only.

\section{Discussion}

Both, arthroscopic lavage and arthroscopic lavage and debridement, are relatively conservative salvage approach to moderately degenerative knee in old population. There is a controversy if these treatments are advantageous to conservative treatment or to more extensive treatment by prosthetic arthroplasty in older population.

There are reports that might support this conservative treatment, e.g. McLaren et al. found 65\% improvement of symptoms after arthroscopic debridement of arthritic knees after short, average 2 years, follow-up [10], Gross et al. found $72 \%$ good results at an average of 2 years after the arthroscopic debridement of arthritic knee [11], Bonamo et al. found 83\% patient satisfaction at 3.3 years follow-up after arthroscopic debridement in younger population [6], Olgilvie-Harris and Fitsialos found $68 \%$ of patient satisfaction after two years and $53 \%$ after 


\section{OPERATED KNEE FUNCTION AT SIX}

MONTHS POST-OPERATIVELY

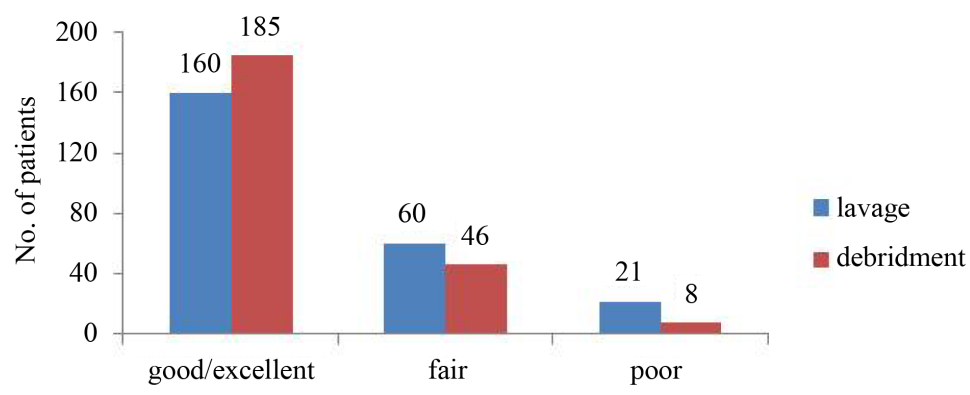

knee function according to HSS

Figure 2. Most of the patients had good and excellent functional results following lavage only and combined debridement and lavage treatments on short term follow up. Significantly better outcome is observed following the latter treatment $(\mathrm{p}<0.01)$.

\section{OPERATED KNEE FUNCTION AT THREE \\ YEARS POST-OPERATIVELY}

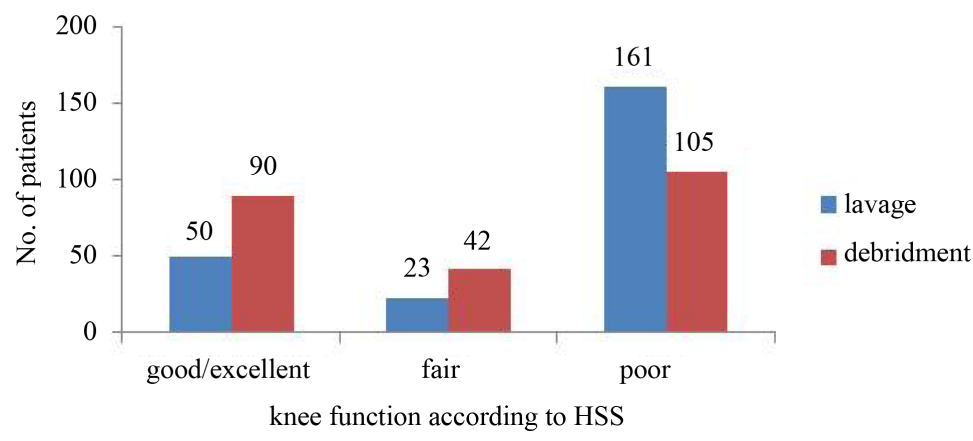

Figure 3. Deterioration in the rate of good and excellent functional results following lavage only and combined debridement and lavage treatments on midterm follow up. Significantly better outcome is observed following the latter treatment $(\mathrm{p}<0.001)$.

\section{OPERATED KNEE FUNCTION AT SIX \\ YEARS POST-OPERATIVELY}

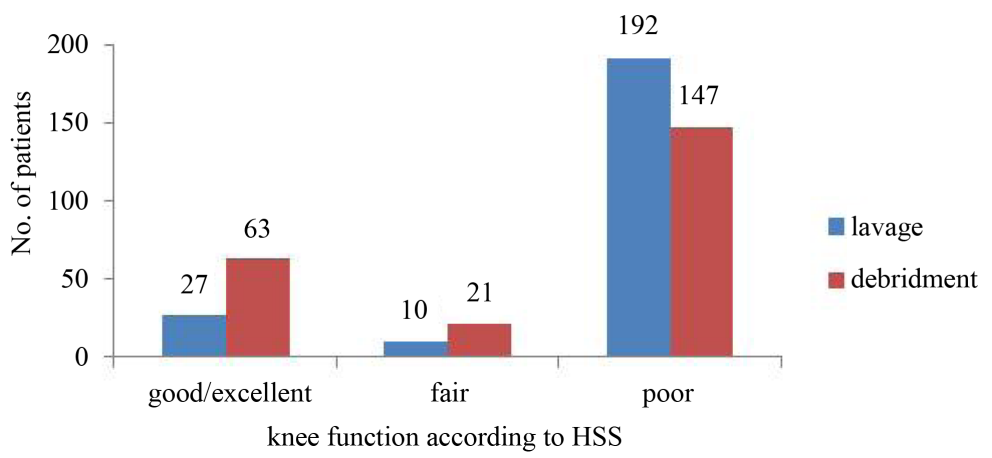

Figure 4. Most of the patients had poor functional results following lavage only and combined debridement and lavage treatments on long term follow up. But significantly better outcome is observed following the latter treatment $(p<0.001)$. 
KNEE FUNCTION FOLLOWING TWO TYPES OF

SURGERY AT FOLLOW UP

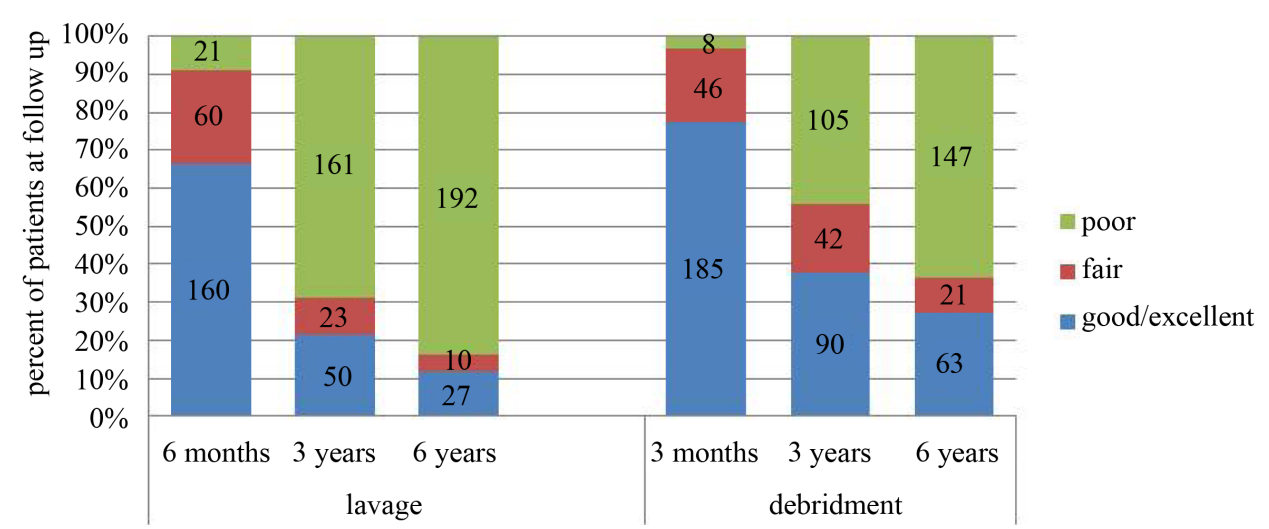

Figure 5. Summarized representation of the outcome data following arthroscopic treatment of arthritic knee by lavage only and by combination of lavage and debridement. Gradual deterioration of the operated knee from short term to long term follow up is evident following both treatments, but in larger extent following the lavage only treatment $(\mathrm{p}<0.001)$.

four years' follow up following arthroscopic debridement of degenerative knees [7].

In the present large scale study we confirm relatively satisfactory results at midterm follow up (3 years) following debridement and lavage of moderately arthritic knees but on the long term follow up (6 years) we observed a significant deterioration of knee function in these patients. Additionally the treatments by arthroscopic lavage only showed unsatisfactory results even after midterm follow up. Therefore we show that arthroscopic lavage only treatment should not be suggested to patients that expect at least midterm improvement in symptoms.

Additionally we can conclude from our results that only a selected group of patients with expectation for short term (of several months) relief of knee symptoms might benefit from arthroscopic knee lavage with a benefit of low surgical morbidity.

For the patients with higher demands and expectations for long term rehabilitation and improvement both arthroscopic treatments are not satisfactory in more of $60 \%$ of cases.

The presented data in a large series of patients provide an important tool in the decision making and tailoring of the surgical procedure for the patients with moderate symptomatic OA of knee, who have different expectations from surgical treatment on short, midterm and long term period of time.

\section{References}

[1] Bert, J.M. and Gasser, S.I. (2002) Approach to the Osteoarthritic Knee in the Aging Athlete: Debridement to Osteotomy. Arthroscopy, 18, 107-110. http://dx.doi.org/10.1053/jars.2002.36513

[2] Bjordal, J.M., Ljunggren, A.E., Klovning, A., and Slørdal, L. (2004) Non-Steroidal Anti-Inflammatory Drugs, Including Cyclo-Oxygenase-2 Inhibitors, in Osteoarthritic Knee Pain: Meta-Analysis of Randomised Placebo Controlled Trials. British Medical Journal, 329, 1317-1322.

[3] Bradley, J.D., et al. (1991) Comparison of NSAIDs in the Treatment of Osteoarthritis of the Knee. The New England Journal of Medicine, 325, 87-92. http://dx.doi.org/10.1056/NEJM199107113250203

[4] Divine, J.G., and Shaffer, M.D. (2011) Use of Viscosupplementation for Knee Osteoarthritis: An Update. Current Sports Medicine Reports, 10, 279-284.

[5] Harwin, S.F. (1999) Arthroscopic Debridement for Osteoarthritis of the Knee: Predictors of Patient Satisfaction. Arthroscopy: The Journal of Arthroscopic \& Related Surgery, 15, 142-146. http://dx.doi.org/10.1053/ar.1999.v15.015014

[6] Bonamo, J.J., Kessler, K.J. and Noah, J. (1992) Arthroscopic Menisectomy in Patients over the age of 40. The American Journal of Sports Medicine, 20, 422-428. http://dx.doi.org/10.1177/036354659202000410

[7] Ogilvie-Harris, D.J. and Fitsialos, D.P. (1991) Arthroscopic Management of the Degenerative Knee. Arthroscopy: The Journal of Arthroscopic \& Related Surgery, 7, 151-157. http://dx.doi.org/10.1016/0749-8063(91)90101-3

[8] Ranawat, C.S., Insall, J. and Shine, J. (1976) Duo-Condylar Knee Arthroplasty: Hospital for Special Surgery Design. Clinical Orthopaedics and Related Research, 120, 76-82. http://dx.doi.org/10.1097/00003086-197610000-00012 
[9] Bourke, G.J. and Daly, L.E. (1985) Interpretation and Uses of Medical Statistics. 3rd Edition, Blackwell Scientific Publication, Oxford, 299-300.

[10] McLaren, A.C., Blokkerb, C.P., Fowler, P.J., Roth, J.N. and Rock, M.G. (1991) Arthroscopic Debridement of the Knee for Osteoarthritis. Canadian Journal of Surgery, 34, 595-598.

[11] Gross, D.E., Brenner, S.L., Esformes, I. and Gross, M.L. (1991) Arthroscopic Treatment of Degenerative Joint Disease of the Knee. Orthopedics, 14, 1317-1321. 